\title{
Gênese do conhecimento em oncologia: produção e difusão da informação técnica e científica entre profissionais especialistas da ár ea assistencial em cuidados paliativos do I nstituto Nacional de Câncer no Brasil. R io de J aneiro
}

Genesis of knowledge in Oncology: production and diffusion of technical and scientific information among specialist professionals in the assistance area on palliative care at the Instituto $\mathrm{Nacional}$ de Câncer in Brasil. Rio de J aneiro

Autor: Pedro Luiz Fernandes

Orientadora: Prof. a Dra Rosali Fernandez de Souza

\section{Resumo}

0 presente trabalho investiga como os processos de produção e difusão da informação técnica e científica ocorrem entre os profissionais especialistas da área assistencial, em cuidados paliativos na construção do conhecimento em O ncologia. 0 principal interesse é conhecer necessidades e usos de informação dos especialistas e entender como isto se relaciona com o conhecimento e com os meios de comunicação disponíveis, isto é, como este compartilhamento é visto em termos da transferência de informação visando à geração do conhecimento. 0 conceito de informação foi relacionado ao contexto da cultura institucional para permitir um melhor entendimento de como as práticas sociais entre os profissionais em cuidados paliativos influenciam na troca de informação e conhecimento. 0 estudo foi desenvolvido em duas etapas: a primeira consistiu no delineamento do comportamento informacional dos especialistas do IN CA e a segunda descreveu como é feita a transferência de informação. Os resultados mostraram uma interação significativa entre eles quanto à solução de problemas nas atividades rotineiras de suas práticas assistenciais. O s especialistas desenvolveram uma eficiente rede de comunicação para obter e difundir informação visando à produção do conhecimento na solução de problemas específicos. Os resultados também mostraram que existe um hiato entre a comunicação informal e formal da informação técnica e científica originária das práticas assistenciais. Este hiato necessita ser melhor entendido em bases institucionais visando a produção de conhecimento em O ncologia.

Tese de D outorado apresentada ao Instituto Brasileiro de Informação em Ciência e Tecnologia (M CT/IBICT), em 2004 Patrocínio do Conselho Nacional de D esenvolvimento Científico e Tecnológico - CN Pq 